\title{
Disseminated Fusariosis Presenting with Pulmonary Nodules Following a Line Infection
}

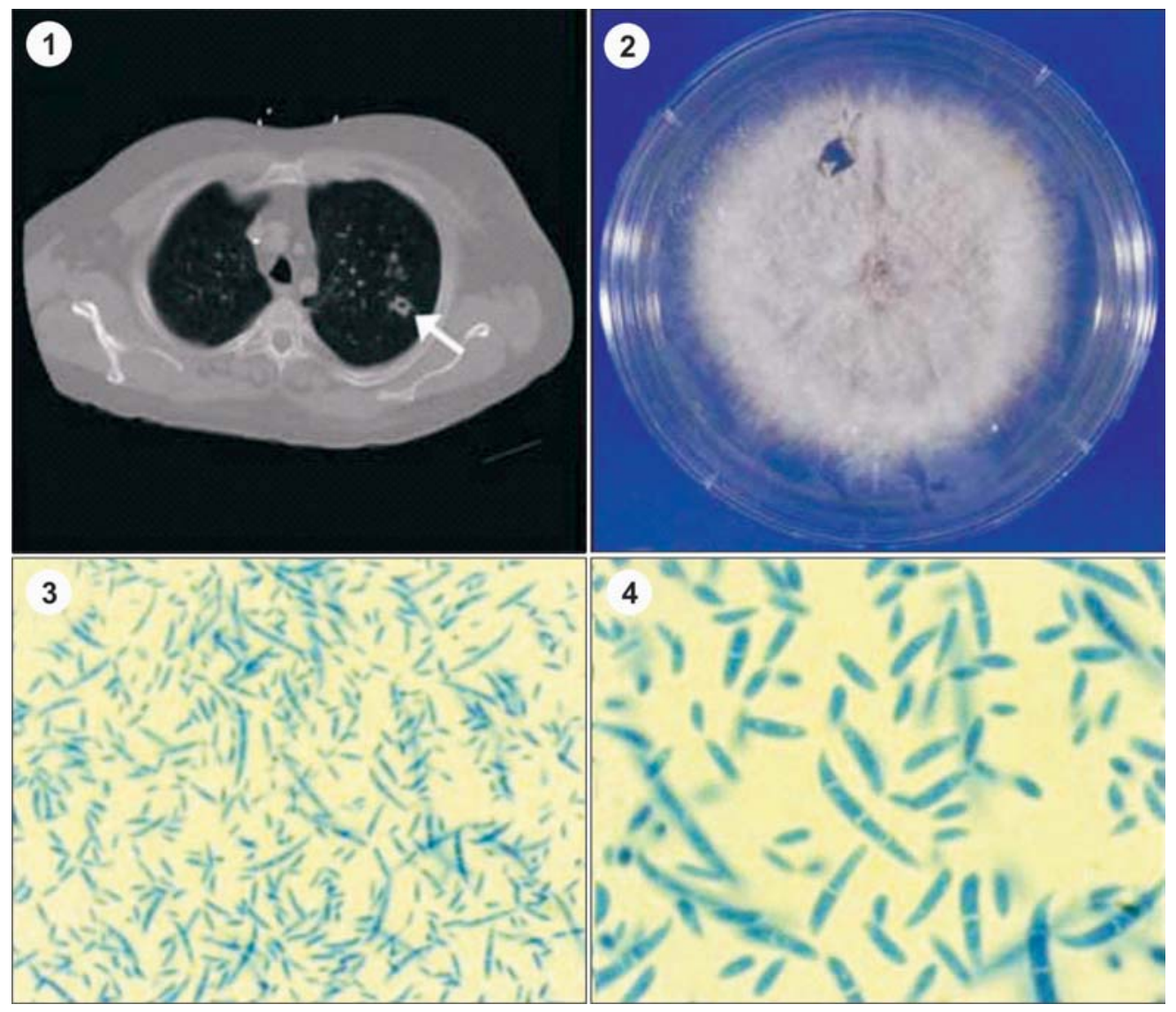

Figure 1. Bilateral pulmonary lesion, some with cavitation (arrow) were present on a thoracic CT scan. Figure 2. Sabouraud dextrose agar plate. showing the typical white, cottony, colony morphology of the Fusarium sp. Figures 3 and 4: Tease prep with lactophenol aniline blue stain demonstrating shorter, simple microconidia and the sickled, multiseptated, macroconidia, characteristic of Fusarium spp.

A 54 year-old male with severe emphysema steroiddependent was admitted to the hospital with perforated diverticulitis. Ten days following surgery and placement of a central venous catheter for antibiotic administration, the patient developed fever, chills, dyspnea, and nonproductive cough. Imaging studies demonstrated bilateral pulmonary nodules, including some with cavitation (Figure 1). Blood cultures grew a mold, which was later identified as Fusarium sp. (Figures 2-4). The patient's central line was removed and he was treated with intravenous voriconazole for 14 days. Cultures of the catheter tip again revealed Fusarium sp. Fusarium spp. are common soil saprophytes that are associated with human infections both locally and systemically. Patients at most risk for disseminated fusariosis are those who are immunosuppressed, including those with neutropenia and patients being treated with corticosteroids or other immunosuppressive agents. The presentation is usually a disseminated infection with persistent fever despite antimicrobial therapy, and with widespread skin lesions. On the other hand, fusariosis in immunocompetent individuals is unusual, but risk factors include tissue breakdown, such as trauma or the presence of a foreign body (i.e. contact lenses, catheters, peritoneal dialysis). Fusarium spp. can be detected by blood cultures in up to half of patients, whereas other molds, such as Aspergillus spp., are less commonly isolated. Fusarium spp. are very resistant to antifungal agents, and the mortality is significantly higher in immunosupressed patients. In less immunocompromised patients, such as the one we present here, removal of the foreign object and treatment with an antifungal agent (voriconazole or amphotericin) will usually lead to successful recovery.

\section{Madariaga, Miguel G, MD and Kohl, Shane, MD University of Nebraska Medical Center}

The Brazilian Journal of Infectious Diseases 2006;10(6):426. ㄷ 2006 by The Brazilian Journal of Infectious Diseases and Contexto Publishing. All rights reserved. 